\title{
33 The Olympic and Paralympic Games as a technology showcase
}

Franz Waldenberger

With the world watching, the Olympic Games provide an ideal case to demonstrate national prowess, not only in the field of sports. When Japan hosted its 1964 Olympics, the Shinkansen, the world's first high-speed rail system, had just started operation. The 1964 Games were the first to be broadcasted via satellites and the first using electronic time measurement, a technology developed and deployed by the Japanese precision instrument company Seiko. These showcases of innovation and technological leadership demonstrated Japan's ambitions and potential, and within less than two decades, the country established itself as a leading high-tech nation.

Today, Japan is one of the most research-and-development intensive economies. The country tops international patent statistics and boasts one of the largest technology-trade surpluses. It was in Japan where the first mobile internet services were successfully launched in 1999. In 2013, the year Japan won the bid for the Olympics, the government officially declared to make Japan the world's most advanced IT nation. In 2016, this goal was topped by the vision to transform Japan into a "super smart" society and to lead the world into the next stage of human civilization, termed "Society 5.0."

Japan aims to use the Olympics to underline and showcase its ambitions as a frontrunner in the digital transformation. Tokyo 2020 is to be remembered as the "smart" Games. The $5 \mathrm{G}$ mobile communication network is to be deployed in time for the events to allow live-streaming and viewing systems using $3 \mathrm{D}$ and $360^{\circ}$ technology. The national broadcasting company NHK plans to air events in ultra-high $8 \mathrm{~K}$ resolution quality. Driverless vehicles will demonstrate Japan's advancements in the field of autonomous driving. At many locations, robots will provide spectators with information or serve refreshments. Japan also plans to digitally augment its worldrenown hospitality with devices and applications offering automatic instantaneous translation. Surveillance technology including drones and state-of-the-art facial recognition software is to ensure the security and smooth operation of the Games.

There had also been news that the official fireworks at the opening and closing ceremonies might be replaced by man-made meteor showers, a technology developed by a Tokyo-based high-tech startup. However, that product will likely not be ready in time for the Games.... 